\title{
Histopathological Studies on the Cystic Formation of the Human Urothelium
}

\author{
SHINSHI NODA AND KOSAKU ETO \\ Department of Urology, Kurume University School of Medicine, Kurume, 830 Japan
}

Received for Publication January 17, 1990

\begin{abstract}
Summay: Histopathological examination of pyeloureteritis cystica and cystitis cystica revealed the mechanisms for the cystic formation of the urothelium. Chronic stimulation with inflammation or physical stimulation with crystals or calculi causes the urothelium to form an inflammatory crypt. The crypt is isolated as a result of an adhesive occlusion of the urothelium at the orifice of the crypt. This crypt is an immature cyst that cannot be clinically detected. A von Brunn's cell nest represents a cut surface of the immature cyst. The inflammatory cyst isolated from the urinary tract, i.e., the immature cyst, gradually grows into a complete cyst, i.e., a clinically visible mature cyst, because of a hydrodynamic flow between the surrounding tissue and neogenetic capillaries, and inflammation. These findings indicate that von Brunn's cell nest, and glandular and cystic formation, occur during development from an inflammatory crypt to an immature cyst and then, a mature cyst.
\end{abstract}

Key words: cystitis cystica - pyeloureteritis cystica - urothelium - metaplastic cystitis - cystitis glandularis

\section{Introduction}

The lumen of the urinary tract, through which the urine passes, is covered with transitional epithelium, except for part of the urethra. This transitional epithelium develops inflammatory changes in response to various types of stimulation, and when these changes become chronic, epithelial metaplasia and hyperplastic lesions of the submucosal layer occur. One metaplastic lesion is the cystic formation of the transitional epithelium, which clinically assumes the form of cystitis cystica or pyeloureteritis cystica. Evidence indicates that the cystic formation of the transitional epithelium is related to prolonged stimulation from chronic urinary tract infections or foreign bodies (calculi, crystals) in the urinary tract. However, there is no accepted theory for the mechanism of cystic forma- tion. Brunn's view of epithelial cell nests is widely supported, but has been challenged by some research groups. To clarify the pathophysiology of cystitis cystica, the mechanism of cystic formation was analyzed by light and electron microscopy.

\section{Experimental Methods and Materials}

Tissue blocks including cysts were surgically resected from patients diagnosed clinically as having pyeloureteritis cystica or cystitis cystica for preparation as light and electron microscopic specimens. The tissue blocks were fixed in formalin, embedded in paraffin and stained with hematoxylin and eosin $(\mathrm{H}-\mathrm{E})$ for examination under a light microscope. Part of each tissue block was minced into $1 \mathrm{~mm}^{3}$ fragments, which were prefixed with cacodylate-buffered $2.5 \%$ glutaral- 


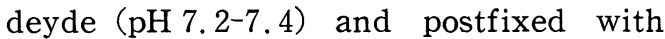
buffered osmic acid $\left(0-4^{\circ} \mathrm{C}\right)$ for $60 \mathrm{~min}$. Postfixed tissue fragments were dehydrated with an ethanol series, treated with propylene oxide and embedded in Epon 812. Embedded fragments were cut thinly and double stained with uranyl acetate and lead hydroxide for electron microscopic examination.

\section{Results}

\section{Macroscopic Findings}

Cysts of the vesical and pyeloureteral mucosa were examined by cystoscopy or grossly at surgery. They were hemispheric protuberances, 1-4 $\mathrm{mm}$ in diameter, containing fluids that differed in cystoscopic transmittance from translucency to turbidity. There were no capillaries at the top of the cysts, but there was a circular (ring-shaped) neogenetic vascular network surrounding each cyst (Fig. 1).

\section{Light Microscopic Findings}

Serial sections were prepared and stained with $\mathrm{H}-\mathrm{E}$ for examination (Fig. 2 ). The cystic structure below the ureteral mucosa in (1)-A was a crypt in communication with the ureteral lumen in (2) and (3) and again was a cystic structure in (4). Likewise, the structure in (1)-B was a crypt in continuity with the urothelium, but was a cystic structure below the ureteral mucosa without continuity with urothelium in (2), (3) and (4). These findings indicate that (1)-A and (1)-B were crypts caused by depressions of the urothelium, which is supported by the absence of fluid in the crypts. The cystic structures, other than $A$ and $B$, showed no continuity with the urothelium; but contained fluids with different $\mathrm{H}-\mathrm{E}$ staining properties. Therefore, the cystic structures could represent the cyst with clinical cystoscopic differences in transmittance. Cystoscopy re- vealed well-developed capillaries around the cysts (Fig. 1-(1), and histologically, these capillaries contained erythrocytes in the space between the thin cyst walls (Fig. 3). The cyst wall consisted only of the lowermost cell layer in contact with the basal plate of the transitional epithelium, as the nucleus and cytoplasm were completely flat. When crypt " $B$ " in Fig. 2 was followed in serial sections, the stump formed a complete cell nest. The cell nest had the morphology of superficial cells of the transitional epithelium at the center and that of cells of the deep basal layer at the periphery (Figs. 4, 5 and 6).

Thorough examination of the cysts that contained fluids showed that the superficial cells in the cyst wall were cylindrical and edematous to exfoliate (Figs. 7 and 8 ). There were cysts filled with edematous exfoliated cell debris, that had cell membranes and nuclei (Fig. 9). In the cysts filled with urine components, the cell debris appeared as a homogeneous secretion with differences in $\mathrm{H}-\mathrm{E}$ staining (Fig. 2).

\section{Electron Microscopic Findings}

Under the electron microscope, the cell nest (Fig. 6), which was completely isolated below the ureteral membrane and appeared unrelated to the urothelium, had the morphological features and architecture of the urothelium. Centered around the lumen, cells were in contact with each other through slender microvilli that interdigitated like a hinge and were connected by complex desmosomes. These cells had very coarse and irregular intercellular spaces. In the cytoplasm, small mitochondria, rough endoplasmic reticuli and Golgi apparatuses were present, but they were immature and small few in number. Tonofilaments were also observed and appeared to be fairly well preserved. The nucleus was long, oval-shaped, without marked embayment; and the nucleoli 
were obvious. Intercellular migration of polymorphonuclear leukocytes (PL) was frequently observed (Fig. 10). There were two types of cells in the isolated cyst (immature cyst) that had lost continuity with the urinary tract, just previously. The first type was cells that had herniated into the cystic lumen. The cytoplasm in the herniated portion contained only a few organelles, with no microvilli on the cell surface. These cells were exfoliated to become cellular debris in the cystic lumen (Fig. 11). The second type was cells that were markedly compressed to become flat, containing only a very few organelles. Superficial epithelial cells had only a few microvilli and a few fusiform vesicles with an asymmetric unit membrane specific for the transitional epithelium, probably due to the compression and extension. In the intercellular space, $\mathrm{mi}$ crovilli were entangled like a hinge to make contact with each other (Fig. 12). The epithelial cells of these cysts exfoliated into teh lumen in a stepwise manner, starting with the superficial layer, so that only the lowermost layer of cells were present, as was observed by light microscopy (Fig. 3 ). These cells were flat in a zonal form and formed a mature cyst wall together with the basal plate. Outside the cyst, there was a large volume of minute granular substances with an appearance like interstitial fluid, in which there was a newly developed blood vessel containing erythrocytes (Fig. 13). The cyst was filled with minute granules, $50-150 \mathrm{~nm}$ in diameter, containing degenerative organelles and nuclei. Furthermore there was a homogenous region, as a result of liquefaction of the organelles and nucleus (Fig. 14).

\section{Discussion}

It has been well established that cystitis cystica and pyeloureteritis crystica are tissue responses to chronic stimulation of the ureteral mucosa with inflammation, or physical stimulation of the mucosa by crytstals or calculi. However, there is no accepted theory with respect to the cystic formation under these conditions, and the only currently accepted view is Brunn's epithelial cell nests. In his view, the urothelium grows downward in response to chronic stimulation to form epithelial cell nests below the mucosa. These nests undergo central necrosis to form a cyst. Stirling and Ash (1947) challenged the view by arguing that epithelial cell nests are nothing but a morphological feature of the cut surface of the tissue and are in continuity with the epithelial cells in serial sections, and that the lumen was produced by an epithelial crypt, rather than by degeneration. Tsuji et al. (1951) implanted human ureteral stones in to the rabbit bladder and histologically examined changes in the urinary bladder. Brunn's view of epithelial cell nests was not supported by several findings. Chronic inflammation occurred in the vesical mucosa, and the mucosal epithelium grew in a cryptic form. The crypt bifurcated and divided into tufts, some of which formed a cyst to induce cystitis cystica. The hyperplastic region of epithelium is considered to show capillary hyperplasia, producing a sufficient blood supply, so that central necrosis is unlikely to occur. However, the possibility (Stirling and Ash, 1947) that crypt-forming cells acquire latent secretory function is also unlikely.

Why are inflammatory crypts formed? Inflammation is a reaction of living tissue to injury. This reaction consists of a series of changes that occur in the terminal vascular floor, blood and connective tissue, and is thought to function to eliminate the cause of the injury and to repair the injured tissue. Therefore, the crypt formation is thought to be a defense response of the urothelium injured by 
chronic stimulation to further stimulation and injuries that are expected to occur. Under the light microscope the crypt was occluded by inflammation to form small cysts. However, the mechanism by which a small cyst subsequently grows into a grossly visible cyst, about $3 \mathrm{~mm}$ in $\mathrm{di}$ ameter, has been explained on the basis of the onset of central necrosis (Fragestrom, 1948) or acquisition of secretory function (Stirling and Ash, 1947), neither of which is persuasive. A study using electron and immunofluorescence microscopes detected secretory-type granules, IgA and IgM, in the epithelial cells on the luminal surface of the cysts associated with cystitis cystica and reported regional secretion of $\operatorname{Ig} \mathrm{A}$ and $\operatorname{IgM}$ and active metabolism of the epithelial cells of the cysts (Walther et al. 1987). IgA secretion is attributable to local immunity for cystitis cystica induced by inflammatory stimulation. Capillary hyperplasia observed around the cyst with the cystoscope and microscope occurs naturally at the site of inflammation (Figs. 1, 3 and 13). At the inflammatory site, vascular permeability was enhanced, showing leakage of plasma protein and migration of the cell components of primarily PL (Figs. 10 and 13). In such an environment, it is difficult to anticipate the changes in an inflammatory crypt, isolated from the urinary tract because of inflammatory occlusion?

The normal urinary tract, especially the vesical mucosa, is constantly in contact with urine, so that the lumen of a crypt should be filled with urine components during the initial stage. The crypt cells, submucosa and capillary loop are thought to surround the isolated urinary crypt (immature cyst) that has accumulated urine components and is no longer in communication with the urinary tract (Fig. 15).

The urinary osmotic pressure is usually in the range $600-800 \mathrm{mOsm} / \mathrm{L}$, although it varies considerably between 30 and $1200 \mathrm{mOsm} / \mathrm{L}$ depending on the conditions required to preserve humoral homeostasis. However, the plasma osmotic pressure is normally $285-295 \mathrm{mOsm} / \mathrm{L}$, and humoral exchange with the interstitial fluid depends on Stirling's rule (Stirling, 1896). Chemical mediators and a hydrodynamic flow are probably responsible for the humoral transfer in the region with inflammation-induced capillary hyperpermeabillity and edema. In cystitis cystica, there is a pressure gradient between the urine osmotic pressure in the lumen of the centrally located isolate crypt and the plasma concentrations of the surrounding connective tissue and capillaries, transporting the humor into the crypt lumen (Fig. 15-(2)). Herniation, glandular changes, exfoliation of isolated crypt cells demonstrate the humoral flow into the cyst, as well as cellular degeneration due to inflammation (Figs. 7-11 and 14). The cyst becomes filled with the exfoliative and degenerative cell components with time (Fig. 14), and these components undergo gradual liquefaction to become a homogeneous fluid (Fig. 3). As a result, the lumen of the isolated cyst gradually expands, probably until the osmotic pressure gradient comes close to equilibrium, or until the crypt-forming cells are unable to extend further (Figs. 3 and 13). This is the mature cyst that is clinically diagnosed as pyeloureteritis cystica or cystitis cystica. A cystoscopic or macroscopic translucency or turbidity of the cyst would be expected with this type of formation.

By classification according to the type of arrangement, the transitional epithelium is a stratified epithelium, which becomes flat or cubic due to the physiologic dilatation and contraction of the urinary bladder. As the present study has shown, the epithelial cells in the isolated inflammatory crypt are edematous and cylindrical during the initial stage, and progression of the inflammation and hydro- 
dynamic flow elevates the intraluminal pressure, resulting in a cylindrical transformation, herniation, exfoliation, extension and flattening of the cells. It is probable that these phenomena account for the clinical coexistence (Erturk et al. 1988) of von Brunn's nests, cystitis cystica and cystitis glandularis.

\section{References}

ERturk, E., Erturk, E. D. V.M., Sheinfeld, J. and DAvis, R.S. (1988). Metaplastic cystitis complicated with von Brunn nests, cystitis cystica, and intestinal type of glandular metaplasia. Urology 32, 165-167.

Fragestrom, D. P. (1948). Proliferative tumors of the ureter and renal pelvis, with further observations of the significance of "Epithelial cell nests": six case reports. J. Urol. 59, 333356.

Starling, E. H. (1896). On the absorption of fluids from the connective tissue space. J. Physiol. 19, 312-326.

Stirling, W. C. and Ash, J. E. (1947). Chronic proliferative lesions of urinary tract. J. Urol. 45, 342-360.

Tsuji, I., Kuroda, K. and Takase, Y. (1951). Study on the metaplasia of the urothelium (II), An experiment of the introduction of the urinary calculi into the bladder of the rabbit, with special reference to the genesis of cystitis cystica. Jpn. J. Urol. 42, 306-312.

Walther, M. M., Campbell, W. G. JR., O'Brien, D. P., Wheathley, J. K. and Graham, S. D. JR. (1987). Cystitis Cystica: An electron and immunofluorescence microscopic study. J. Urol. 137, 764-768. 

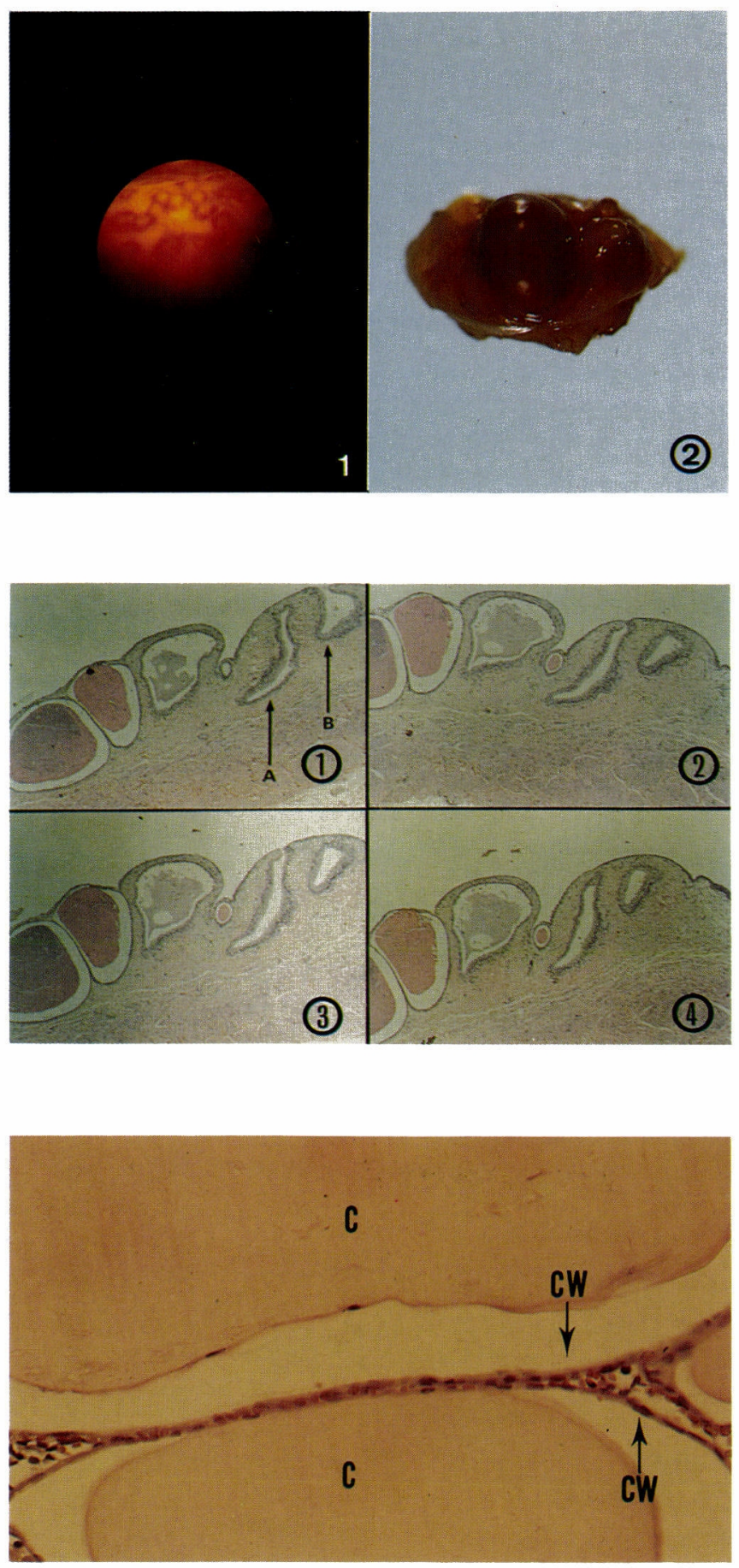

\section{Fig. 1.}

(1) Cystoscopic findings of cystitis cystica. A hemispheric, protuberant cyst about $3 \mathrm{~mm}$ in diameter is surrounded by a ring-shaped neogenetic capillary network.

(2) A specimen obtained at surgery showing translucent cysts of various sizes.

Fig. 2. Serial sections stained with $\mathrm{H}-\mathrm{E}$. A and B are in communication with the urinary tract, and the epithelium is also in continuity with the urothelium. The remaining cysts, which contain fluids different in stainability, are not in communication with the urinary tract. $\times 20$

Fig. 3. The epithelium of cysts consists only of a single flat epithelial layer, and the lumen is filled with a fluid. There are neogenetic capillaries containing erythrocytes between the cysts.

C: cyst, CW: cyst wall. $\times 200$ 


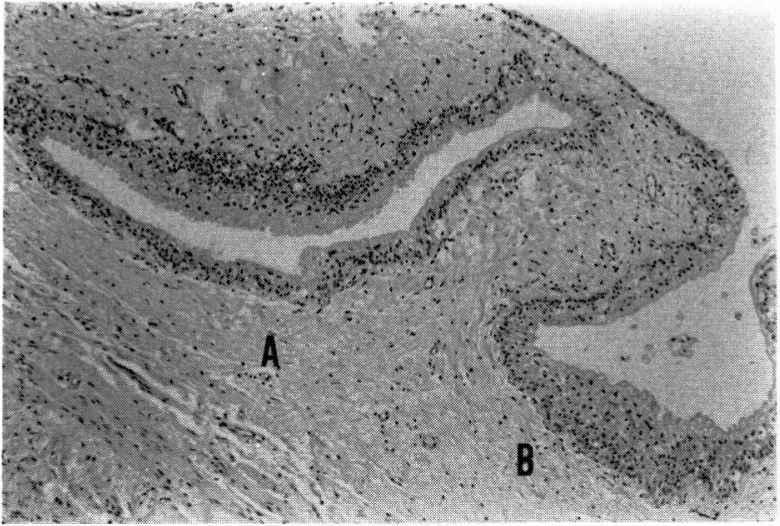

F!g. 4

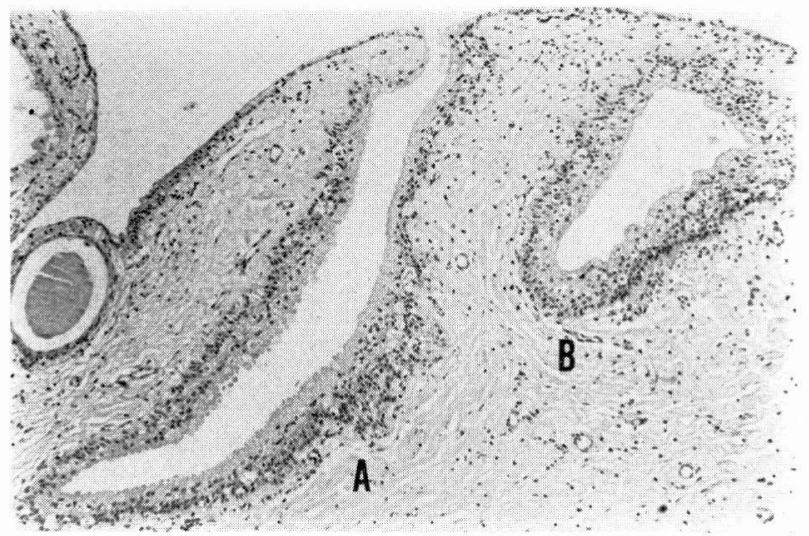

Fig. 5

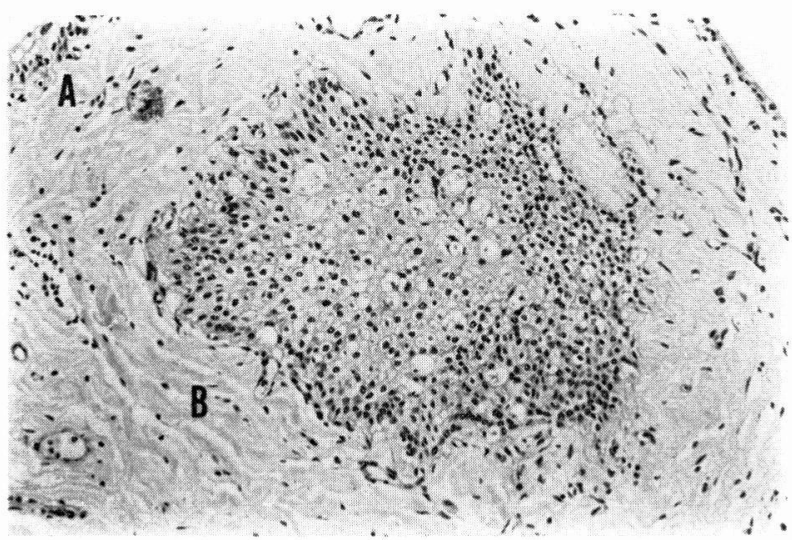

Fig. 6

Fig. 4, 5 and 6. Magnified serial sections of Fig. 2-B. The crypt in communication with the urinary tract(Fig. 4) is an isolated crypt in Fig. 5 and appears like a von Brunn's cell nest in Fig. 6 .

Fig. 4 and 5: $\times 100$. Fig. 6: $\times 200$. 


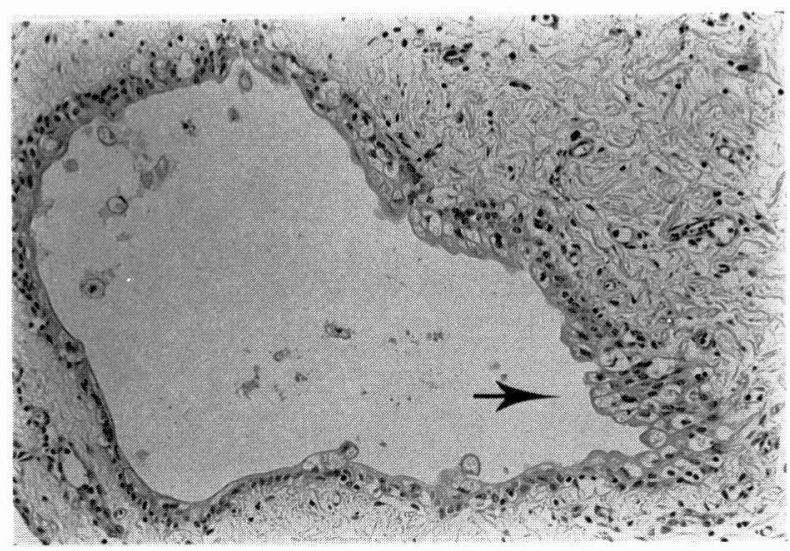

Fig. 7. The epithelium of the isolated crypt shows cells being exfoliated into the lumen and those becoming cubic (arrow mark). $\times 200$

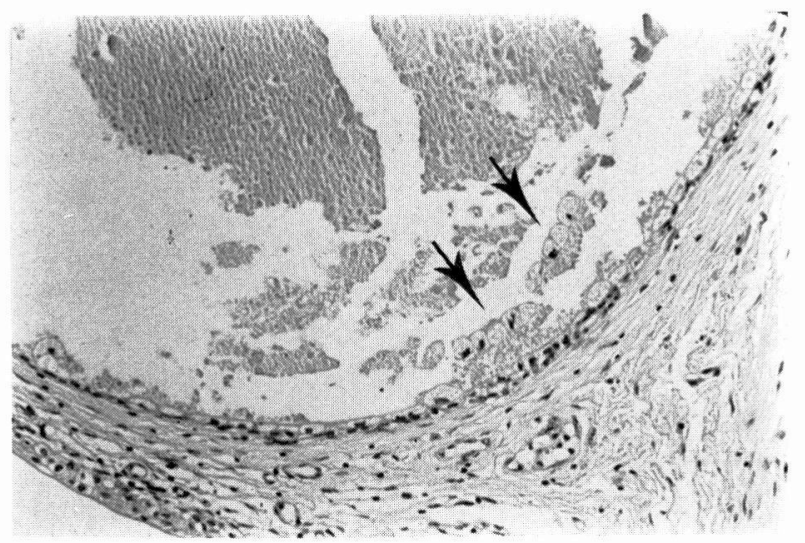

Fig. 8. Edematous epithelial cells (arrow mark) being exfoliated are obvious. $\times 200$

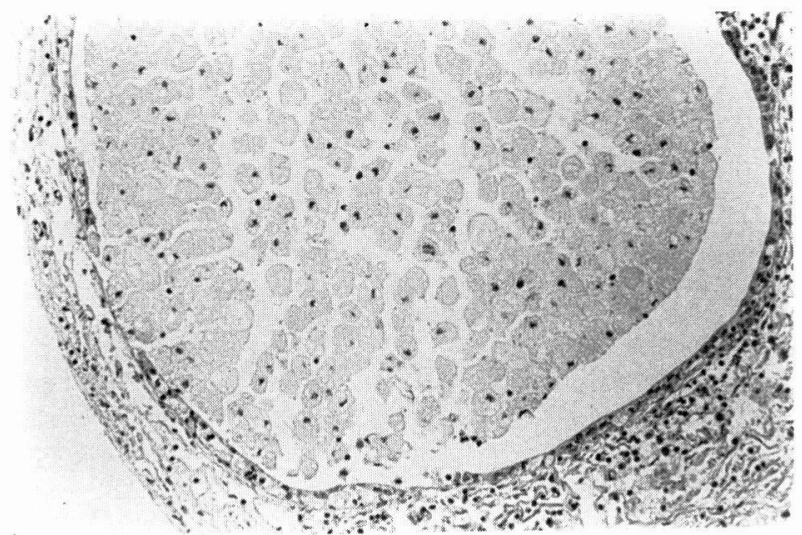

Fig. 9. The lumen of the cyst is filled with degenerative and exfoliative cells, and the cyst wall consists only of a single layer of epithelial cells. $\times 200$ 


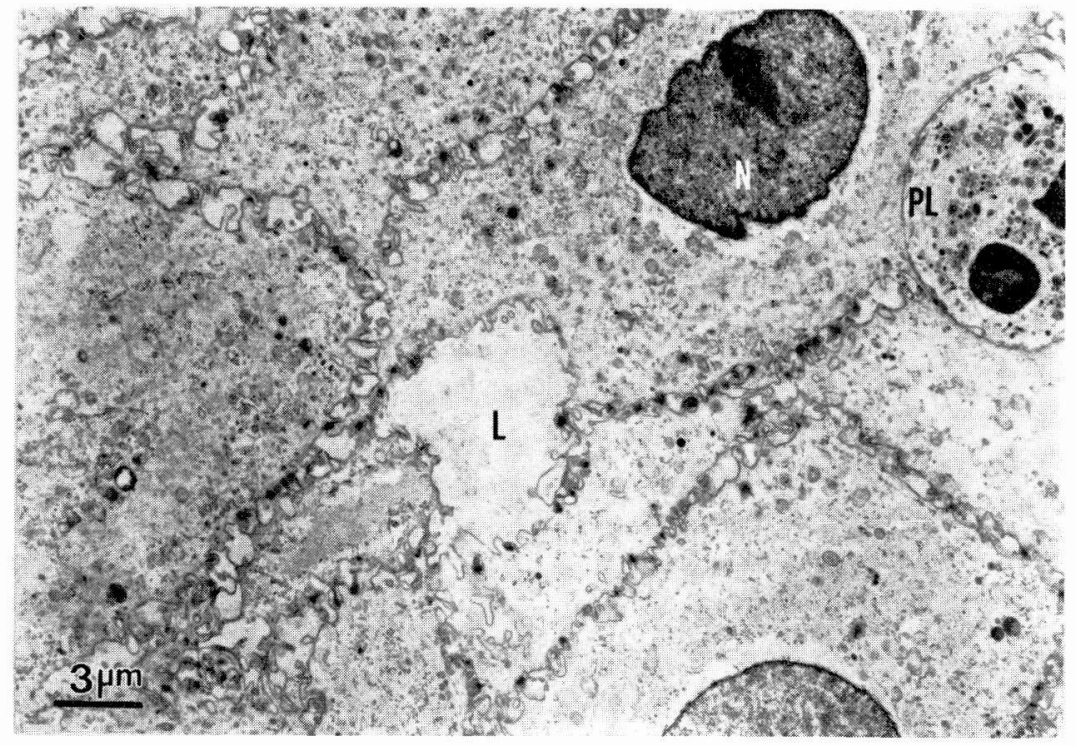

Fig. 10. A von Brunn's cell nest under an electron microscope. A lumen is clearly observed, and cells are in contact with each other through microvilli that are connected in a hinge pattern. The intracytoplasmic architecture shows the morphological features of the transitional epithelium. Migration of PL is observed between cells.

L: lumen, PL: polymorphonuclear leukocyte, $\mathrm{N}$ : nucleus.

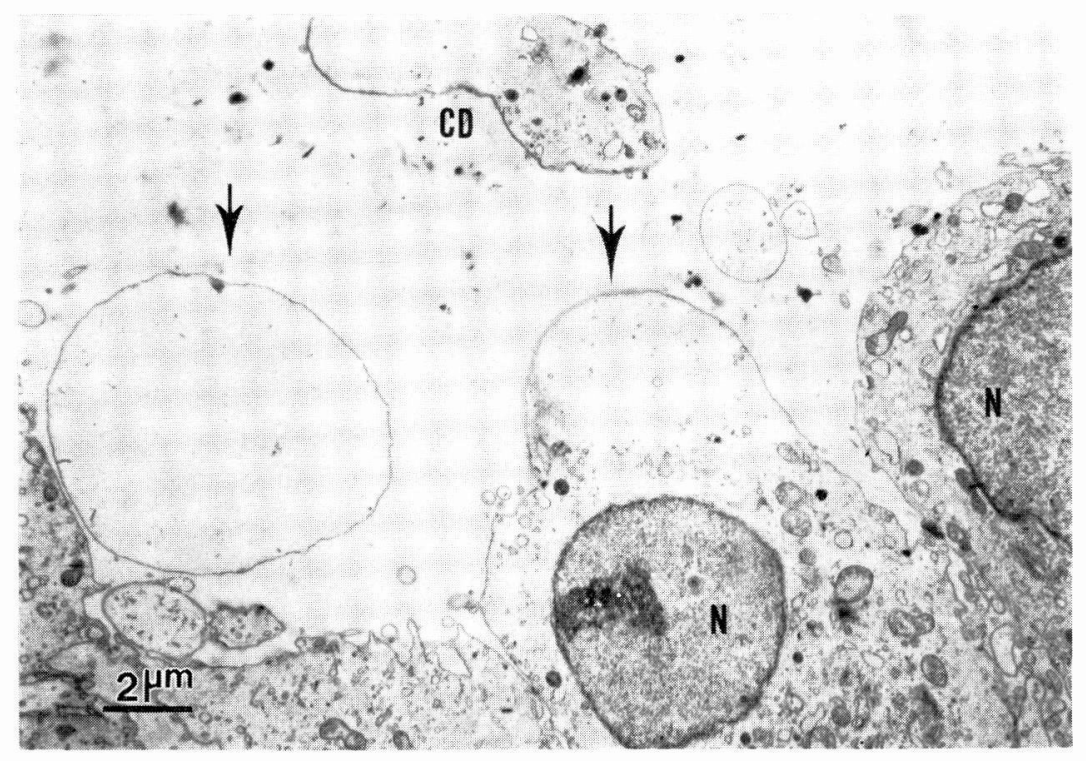

Fig. 11. An electron microscopic photo of cells under herniation (arrow mark) into the cystic lumen and cellular debris in the lumen. The herniating portion contains an extremely small number of organelles.

$\mathrm{N}$ : nucleus, CD: cell debris. 


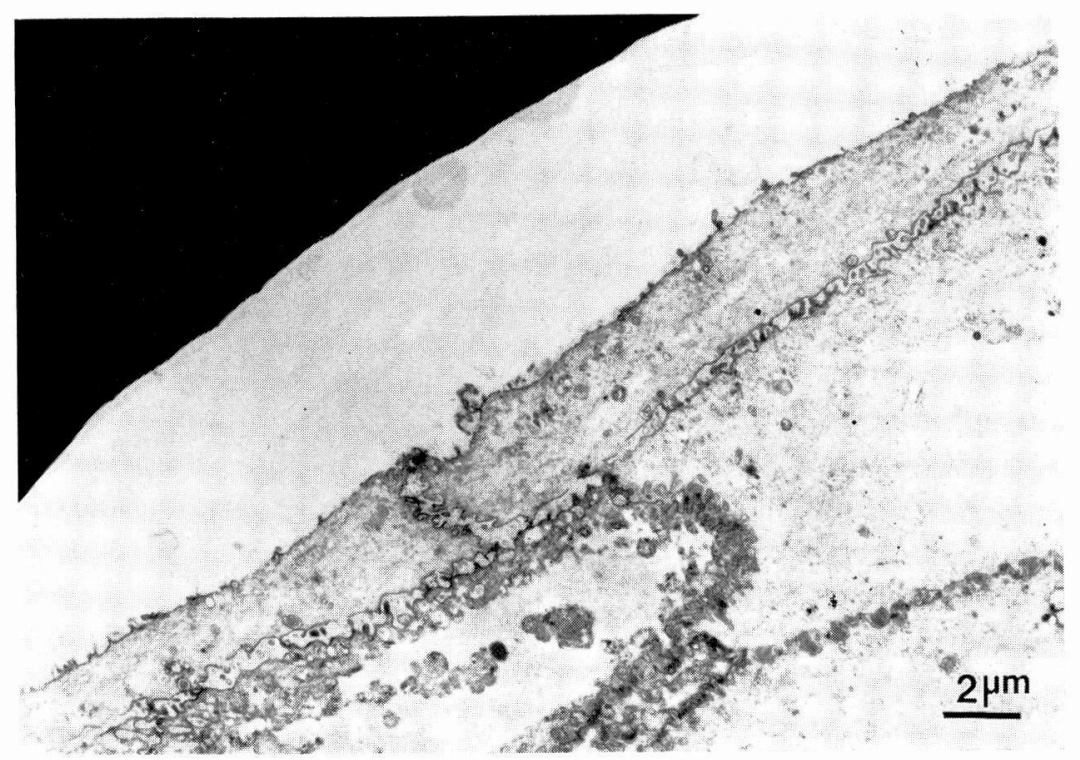

Fig. 12. Compression has flattened and extended the epithelial cells of the cyst and has greatly reduced the number of fusiform vesicles specific for the transitional epithelium.

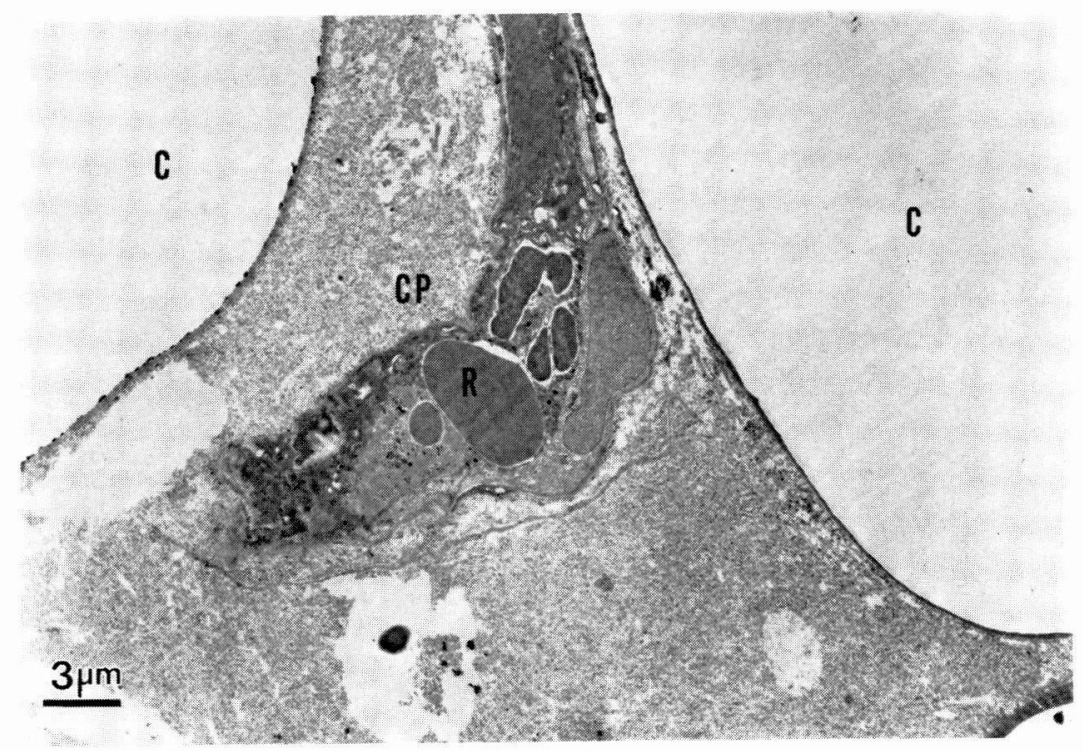

Fig. 13. The completed cyst wall consists only of the lowermost cell layer and is flat with a zonal shape. Neogenetic capillaries are present outside the cyst.

C: cyst, CP: capillary, R: erythrocyte. 


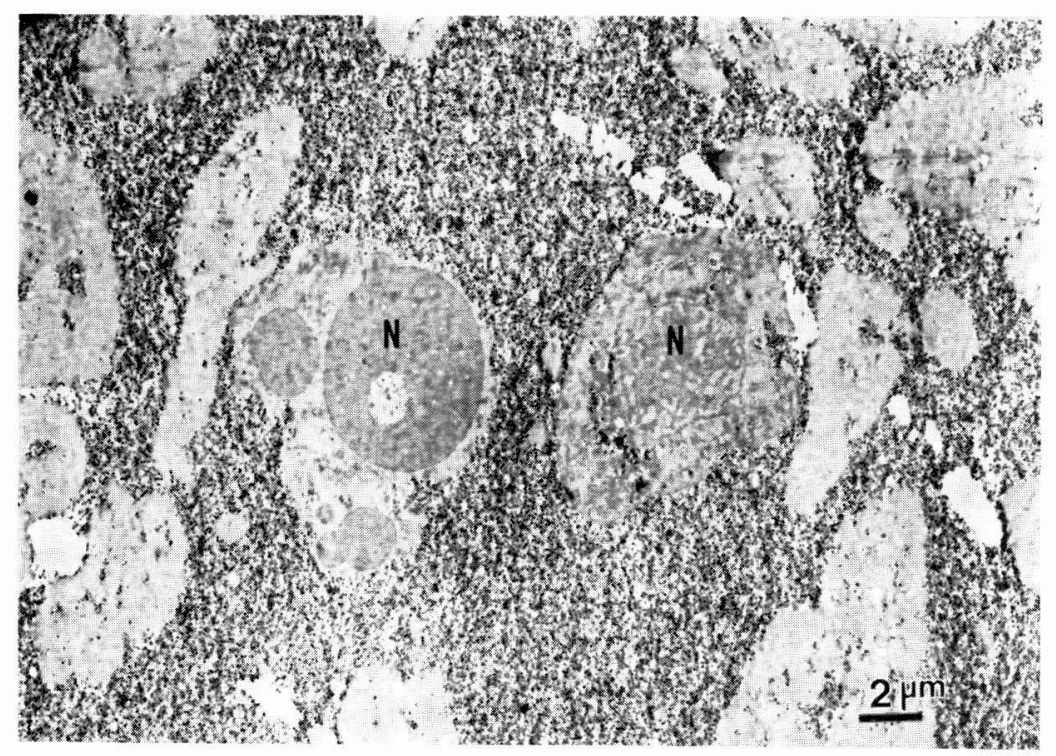

Fig. 14. An electron microscopic image of the substances present in the cyst. Granules, $50-150 \mathrm{~nm}$ in diameter, and degenerative organelles are observed.

$\mathrm{N}$ : nucleus.
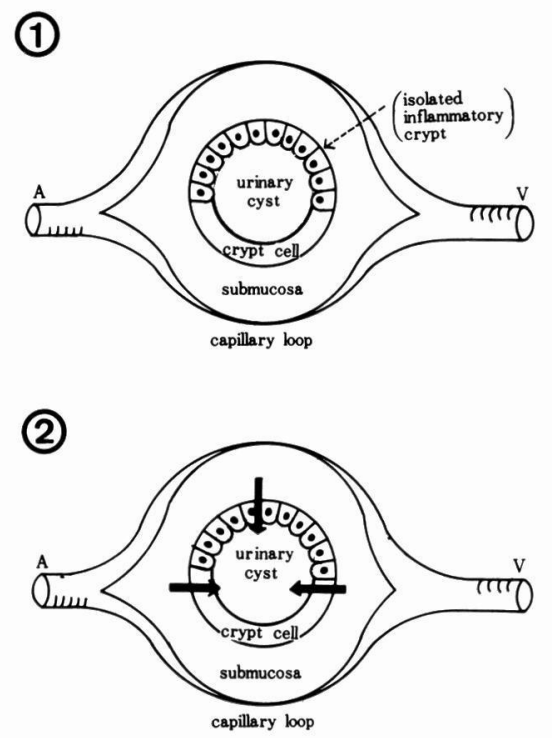

Fig. 15. A scheme for an isolated inflammatory crypt with a crypt cell, submucosa and capillary loop in the center. Local inflammation and a hydrodynamic flow transport the humor in the arrow-marked direction of the arrow. 